\title{
El rendimiento académico en el nivel de educación media como factor asociado al rendimiento académico en la universidad
}

\author{
Academic performance in high school as factor \\ associated to academic performance in college \\ Recibido: 12 de octubre de 2008 - Revisado: 1 de noviembre de 2008 - Aceptado: 6 de noviembre de 2008
}

Angélica María Villalba*

Mileidy Salcedo Barragán**

\section{Resumen}

Esta investigación pretende establecer la relación entre el rendimiento académico en Educación Media y el rendimiento académico en la universidad, enfatizando en el aprovechamiento en Ciencias Naturales y Matemáticas. El estudio es descriptivo correlacional y las variables fueron, el rendimiento académico en Educación Media, los Indicadores de Rendimiento y la Trayectoria escolar. Se determinó la correlación entre las variables a través del coeficiente de correlación de Spearman. Los resultados permitieron determinar que existe una relación positiva entre el rendimiento académico en Educación Media y la Trayectoria Escolar y una relación muy débil entre el rendimiento en Ciencias Naturales y Matemáticas en Educación Media con relación al obtenido en la universidad.

\section{Palabras clave}

Educación Media, Rendimiento Académico, Educación Superior, Indicadores de Rendimiento, Trayectoria Escolar.

\footnotetext{
Abstract

This study intends to find the relationship between academic performance in High School and College, focusing on Natural Sciences and Mathematics. It is a descriptive correlational study, and the variables were academic performance in High School, performance indicators and educational history. The correlations between variables were established with Spearman's correlation coefficient. Results suggest that there is a positive relationship between academic performance in High School and Educational History, and a very weak relationship between performance in Science and Mathematics in High School and performance in College. Key words

Senior High School, Academic Performance, College Education, Performance Indexes, Educational History.
}

\footnotetext{
" Magíster en Educación. Correo electrónico: villalba.angelica@gmail.com

${ }^{* *}$ Magíster en Educación. Correo electrónico: misalba@gmail.com
} 


\section{Introducción}

Una de las preguntas más frecuentes en los estudios relacionados con el rendimiento académico es: ¿el sistema educativo anterior prepara a los estudiantes de forma adecuada para iniciar los estudios universitarios? La alta deserción en los primeros semestres de las diferentes carreras universitarias y el bajo rendimiento obtenido por un gran número de estudiantes (Rodríguez \& Hernández, 2008), parecerían indicar que no. Para las universidades es fundamental el logro de un diagnóstico de las condiciones de sus ingresantes, que le permita hacer unos buenos procesos de selección y mejorar dichas condiciones formulando estrategias de acompañamiento que le permitan al estudiante una vez ingresado no solo mantenerse en el sistema sino también obtener un buen rendimiento académico.

El Colegio El Divino Niño de la ciudad de Santa Marta, es una institución que ha estructurado su plan de estudios otorgándole prioridad desde los primeros años de la educación básica secundaria, al área de Ciencias Naturales y de matemática. En este sentido, se esperaría que las egresadas de esta institución optaran por carreras universitarias con alto contenido en ciencias naturales y además que su rendimiento en las asignaturas relacionadas con esta área fuera altamente satisfactorio. Sin embargo, el colegio no cuenta con referentes distintos a las Pruebas ICFES que le permita evaluar la pertinencia de su currículo y consecuentemente de su plan de estudios, por lo cual el seguimiento de sus egresadas se convierte en una necesidad institucional. El presente estudio tiene como propósito indagar sobre el rendimiento académico de las egresadas del Colegio El Divino Niño en el nivel de Educación Media y su asociación con el rendimiento académico en los programas de pre-grado cursados, especialmente en aquellos con alto contenido en Ciencias Naturales (Ingenierías, Ciencias de la Salud y Ciencias Básicas). El trabajo se realizará con egresadas del Colegio El Divino Niño que cursan programas especialmente en las principales universidades de la ciudad Santa Marta.

Los resultados indican que las egresadas muestran una leve tendencia a mantener en la universidad, el rendimiento académico obtenido en Educación Media, y cuando se hace referencia a las estudiantes que cursan programas relacionadas con las Ciencias Naturales estas presentan en su mayoría trayectorias escolares satisfactorias, sin embargo al analizar aisladamente el rendimiento académico en las asignaturas básicas del programa, éste desciende significativamente, evidenciado en los promedios bajos obtenidos en las asignaturas con alto contenido de ciencias naturales y matemáticas.

\section{Problema de investigación y método}

De la totalidad de las egresadas de El Colegio El Divino Niño entre los años 2002 y 2006 el colegio ha realizado un parcial seguimiento al $85 \%$ de la población total. De acuerdo con estos datos, el porcentaje de éstas, que eligieron programas académicos relacionados con las ciencias naturales es inferior al esperado por la institución. Así mismo los resultados de las estudiantes en las pruebas ICFES en el área de Ciencias Naturales y Matemáticas también son inferiores a los esperados por el colegio, teniendo en cuenta que su formación tiene profundización en estas áreas. Esta situación genera la necesidad de analizar la pertinencia de la propuesta curricular de la institución, y especialmente la preparación académica en educación media, en las áreas de Ciencias Naturales y Matemáticas. Es importante además indagar sobre el rendimiento de las egresadas en los diferentes programas académicos cursados en la universidad, con el propósito de determinar la pertinencia de la formación con profundización en Ciencias Naturales impartida por el colegio así como revisar, replantear y proponer nuevas estrategias que se traduzcan en mejoramiento de la educación ofrecida a las estudiantes. En este orden de ideas, se plantean 
las siguientes preguntas de investigación: ¿Cómo fue el rendimiento académico de las egresadas del Colegio El Divino Niño en el nivel de Educación Media?, ¿Cuál es el rendimiento académico, de las egresadas que cursan programas de pregrado en la universidad?, ¿En qué programas de Educación Superior evidencian mejor rendimiento académico las egresadas del Colegio El Divino Niño?, ¿Existe relación entre el rendimiento académico de las egresadas en su Educación Media y su rendimiento en la universidad? y ¿Existe relación entre el rendimiento académico en Ciencias Naturales y Matemáticas en Educación Media y el rendimiento en la universidad en las asignaturas relacionadas con estas áreas?

\section{Método}

La presente investigación es un estudio descriptivo de tipo correlacional, que pretende recoger información sobre las variables de estudio para detallar las características de las estudiantes que constituyen la muestra, en términos del rendimiento académico obtenido en Educación Media y en la universidad. El estudio tiene como propósito inicialmente describir las variables rendimiento académico en Educación Media, los indicadores de rendimiento y la trayectoria escolar en la universidad. Posteriormente se establecerá el grado de asociación entre las variables Rendimiento Académico en Educación Media y Trayectoria Escolar

La población la constituyen las 247 egresadas de los años 2002-2006, del Colegio El Divino Niño. La muestra la conforman 106 egresadas que cursan programas académicos en diferentes universidades, especialmente de la ciudad de Santa Marta, y que se encuentran matriculadas entre el primer y décimo semestre. Estas estudiantes constituyen el $43 \%$ de la población. La selección de la muestra se realizó a través de un muestreo intencional de tipo no probabilístico, respondiendo a criterios tales como facilidad para el acceso a la información y los costos estimados en términos de tiempo y dinero.

\section{Hipótesis}

Para el presente estudio se consideran dos hipótesis alternas:

Hipótesis 1: Existe correlación estadísticamente positiva entre el rendimiento académico de las egresadas de El Colegio El Divino Niño en Educación Media y su rendimiento académico en la universidad.

Hipótesis 2: Existe correlación estadísticamente positiva entre el Rendimiento Académico en ciencias naturales y matemáticas de las egresadas de El Colegio El Divino Niño en Educación Media y el promedio en las asignaturas relacionadas con estas áreas en la universidad.

\section{Las Variables}

Las variables objeto de estudio son: el rendimiento académico en Educación Media, los indicadores de rendimiento a través de los cuales se construye la trayectoria escolar (Índice de Aprobación en Ordinario IAO, Índice de Promoción IP y el Promedio PROM) y la trayectoria escolar (que determina el rendimiento de las egresadas en la universidad).

Rendimiento Académico en Educación Media

Definición conceptual: el rendimiento académico es un indicador del nivel de aprendizaje alcanzado por el alumno, en tal sentido, el rendimiento académico se convierte en una "tabla imaginaria de medida" para el aprendizaje logrado en el aula, lo que constituye el objetivo central de la educación (Reyes, 2003).

Definición operacional: total de valoraciones académicas obtenidas durante los dos años de Educación Media, de acuerdo con la siguiente escala: 
Tabla 1.

Valoraciones en el nivel de Educación Media

\begin{tabular}{|ll|l|}
\hline \multicolumn{2}{|l|}{ Valoraciones } & \multicolumn{1}{c|}{ Escala } \\
\hline Deficiente (D) & $0-19 \%$ de logros alcanzados \\
\hline Insuficiente (I) & $20-59 \%$ de logros alcanzados \\
\hline Aceptable (A) & $60-79 \%$ de logros alcanzados \\
\hline Sobresaliente (S) & $80-94 \%$ de logros alcanzados \\
\hline Excelente (E) & $95-100 \%$ de logros alcanzados \\
\hline
\end{tabular}

En el plan de estudios del Colegio El Divino Niño se establecen diez áreas fundamentales (Ciencias Naturales, Matemáticas, Humanidades, Educación en Ética y Valores, Educación Religiosa, Educación Física, Filosofía, Ciencias Políticas y Económicas, Informática y Tecnología y Educación Artística), consideradas para cada grado de Educación Media (Artículo 31 Ley General de Educación, 1994). Por lo tanto para determinar el rendimiento alcanzado por las egresadas en este nivel de educación, se consideraron un total de 20 valoraciones. (10 correspondientes a décimo grado y 10 a undécimo) .

Indicadores: para efectos de establecer el nivel de rendimiento alcanzado por las egresadas durante su Educación Media solo se consideraron las valoraciones de excelente y sobresaliente de acuerdo con lo establecido en la siguiente tabla:

Tabla 2.

Nivel de Rendimiento en Educación Media

\begin{tabular}{|l|c|}
\hline $\begin{array}{c}\text { Nivel del } \\
\text { Rendimiento }\end{array}$ & $\begin{array}{c}\text { No. de valoraciones en } \\
\text { Excelente y/o Sobresaliente }\end{array}$ \\
\hline 1=Bajo & $<12 \mathrm{~S} \mathrm{y} / \mathrm{o} \mathrm{E}$ \\
\hline 2=Regular & {$[12,15] \mathrm{S}$ y/o E } \\
\hline 3= Alto & {$[16,20] \mathrm{S} \mathrm{y} / \mathrm{o} \mathrm{E}$} \\
\hline
\end{tabular}

En este estudio, los niveles de regular y alto, se considerarán rendimiento académico satisfactorio.

De acuerdo con los criterios de evaluación establecidos por el Colegio el Divino Niño, valoraciones inferiores al $60 \%$ son consideradas bajas, entre el $60 \%$ y $79 \%$ son aceptables y valoraciones superiores a $80 \%$ se consideran altas. Haciendo la equivalencia correspondiente para el presente estudio, menos de 12 valoraciones en sobresaliente y/o excelente (menos del 60\%), es rendimiento académico bajo, entre 12 y 15 valoraciones sobresalientes $\mathrm{y} / \mathrm{o}$ excelentes $(60 \%$ a $75 \%)$ rendimiento académico regular y de 16 a 20 valoraciones en sobresaliente y/o excelente (80 a 100\%) rendimiento académico alto.

Para determinar el nivel de rendimiento en las áreas de ciencias naturales y matemáticas en Educación Media se tuvieron en cuenta:

1. Solo las valoraciones de aceptable, sobresaliente y excelente definidas en la escala valorativa del Colegio El Divino Niño y

2. las dos valoraciones obtenidas en cada una de estas áreas, correspondientes a los dos grados del nivel de Educación Media, de acuerdo con lo establecido en la siguiente tabla:

Tabla 3.

Nivel de Rendimiento en Ciencias Naturales y Matemáticas en Educación Media

\begin{tabular}{|l|c|}
\hline $\begin{array}{c}\text { Nivel del } \\
\text { Rendimiento }\end{array}$ & $\begin{array}{c}\text { Valoraciones } \\
\text { Obtenidas }\end{array}$ \\
\hline 1=Bajo & $\begin{array}{c}\text { 1A y otra valoración inferior a A } \\
\text { ó 2 valoraciones inferiores a A }\end{array}$ \\
\hline 2=Regular & $\begin{array}{c}\text { 1 valoración S ó E y 1 A } \\
\text { ó 2 valoraciones A }\end{array}$ \\
\hline $3=$ Alto & 2 valoraciones S y/o E \\
\hline
\end{tabular}

Indicadores de rendimiento

Definición conceptual: magnitud numérica referida al rendimiento académico obtenido en los programas de pregrado cursados en la universidad.

Definición operacional: fundamentándose en el trabajo de Chaín, Cruz, Martínez \& Jácome (2003) sobre la relación entre el examen de admisión y la probabilidad de éxito escolar se determinaron tres indicadores de rendimiento: el índice de aprobación en ordinario (IAO), el índice de promoción (IP) y el promedio (PROM). 
Tabla 4.

Definición de Indicadores de Rendimiento

\begin{tabular}{|l|l|}
\hline Indicadores & Definición \\
\hline IAO & $\begin{array}{l}\text { Porcentaje de asignaturas aprobadas en ordinario del total de asignaturas cursadas } \\
\text { (entendiéndose por ordinario el cursar la asignatura por primera vez) }\end{array}$ \\
\hline IP & Porcentaje de asignaturas totales promovidas, del total de asignaturas cursadas. \\
\hline PROM & $\begin{array}{l}\text { Sumatoria de las calificaciones totales dividida entre el número de calificaciones } \\
\text { obtenidas. }\end{array}$ \\
\hline
\end{tabular}

Para el presente estudio se consideró como bajo un promedio inferior a 3,5, como regular los promedios de 3,5 a 3,99 y como altos, promedios de 4,0 a 5,0. Para determinar el nivel rendimiento en la universidad en las áreas de Ciencias Naturales y Matemáticas se estableció el promedio en cada una de ellas a partir de las asignaturas relacionadas con estas áreas del conocimiento.

Indicadores: en esta investigación, para la determinación del IAO y el IP, se respetaron los porcentajes establecidos en la escala determinada por los autores arriba mencionados, sin embargo, para establecer el promedio se hicieron algunas modificaciones, considerando como bajo porcentajes inferiores a 70 (y no a 80 como sugiere la escala original), teniendo en cuenta que en el presente estudio un promedio de 3,5 a 3,99 es considerado regular y su equivalencia en porcentaje está entre el 70\% y el 79.8\% Así mismo se modificaron los porcentajes para los promedios regular y alto.

Tabla 5 .

Indicadores de Rendimiento

\begin{tabular}{|l|c|}
\hline Concepto & Escala \\
\hline Índice de aprobación en ordinario(IAO) & $\begin{array}{c}1=\text { Bajo }<80 \\
2=\text { Regular }[80,<90) \\
3=\text { Alto }[90,100]\end{array}$ \\
\hline Índice de Promoción(IP) & $\begin{array}{c}1=\text { Bajo }<90 \\
2=\text { Regular }[90>,<100) \\
3=\text { Alto }=100\end{array}$ \\
\hline Promedio(PROM) & $\begin{array}{c}1=\text { Bajo }<70 \\
2=\text { Regular }[70,80) \\
3=\text { Alto }[80,100]\end{array}$ \\
\hline
\end{tabular}

Adaptado de Chaín, Cruz, Martínez y Jácome (2003)

\section{Trayectoria Escolar}

Definición conceptual: nivel de rendimiento académico alcanzado a lo largo del programa de pregrado cursado.

Definición operacional: la trayectoria escolar es la categoría en la que se ubica al estudiante, a partir del resultado obtenido de la sumatoria de los valores de los indicadores anteriormente descritos (IAO, IP y PROM)

Indicador: la trayectoria escolar se determinará, de acuerdo con lo establecido en la siguiente tabla: 
Tabla 6.

Indicadores y Categorías

\begin{tabular}{|c|c|c|c|c|c|}
\hline $\begin{array}{l}\text { Código } \\
\text { del estudiante }\end{array}$ & IAO & IP & PROM & IAO+IP+PROM & TRAYECTORIA \\
\hline 001 & 1 bajo & 1 bajo & 1 bajo & 3 & \multirow[t]{3}{*}{$1=$ baja } \\
\hline 002 & 1bajo & 1bajo & 2 regular & 4 & \\
\hline 003 & 1 bajo & 2 regular & 2 regular & 5 & \\
\hline 004 & 2 regular & 2 regular & 2 regular & 6 & \multirow[t]{2}{*}{$2=$ regular } \\
\hline 005 & 2 regular & 2 regular & 3 alto & 7 & \\
\hline 006 & 2 regular & 3 alto & 3 alto & 8 & \multirow[t]{2}{*}{$3=$ alta } \\
\hline 007 & 3 alto & 3 alto & 3 alto & 9 & \\
\hline
\end{tabular}

Tomado de Chaín, Cruz, Martínez y Jácome (2003)

Manejo Estadístico: el valor de la relación de las variables estudiadas se calculó mediante el coeficiente de correlación ñ de Spearman, utilizando el software para tratamiento estadístico SPSS versión 11.5. La magnitud obtenida de la probable relación entre variables fue evaluada atendiendo a la fuerza de la relación y la significación estadística de la misma. Los valores obtenidos cercanos a cero muestran una relación débil, mientras que los que se acercan a +1 o a 1 evidencian una correlación positiva fuerte o una correlación negativa fuerte respectivamente. Lo mismo se corresponde de manera negativa.

\section{Presentación y análisis de resultados}

Para una mejor organización y explicación de los resultados, estos se presentarán respondiendo en su orden, una a una las preguntas de investigación.

\section{¿Cómo fue el rendimiento académico de las egresadas del Colegio El Divino Niño en el nivel de Educación Media?}

La tabla 7 registra el rendimiento académico de las egresadas de El Colegio El Divino Niño, en Educación Media.

Tabla 7.

Rendimiento Académico en Educación Media de egresadas de El Colegio El Divino Niño

\begin{tabular}{|l|l|l|c|c|c|}
\hline \multicolumn{2}{|c|}{} & Frecuencia & Porcentaje & $\begin{array}{l}\text { Porcentaje } \\
\text { válido }\end{array}$ & $\begin{array}{c}\text { Porcentaje } \\
\text { acumulado }\end{array}$ \\
\hline Válidos & Bajo & 14 & 13,2 & 13,2 & 13,2 \\
\cline { 2 - 6 } & Regular & 61 & 57,5 & 57,5 & 70,8 \\
\cline { 2 - 6 } & Alto & 31 & 29,2 & 29,2 & 100,0 \\
\cline { 2 - 6 } & Total & 106 & 100,0 & 100,0 & \\
\hline
\end{tabular}

Los datos obtenidos a partir de los registros académicos de las egresadas en el nivel de Educación Media, muestran un 57,5\% de estudiantes con rendimiento regular, un $29,2 \%$ con rendimiento alto y un $13,2 \%$ con rendimiento bajo. Esto indica que la gran mayoría de estudiantes $(86,7 \%)$ presentó durante el nivel de Educación Media un rendimiento académico satisfactorio (Ver tabla 7). Estos resultados podrían explicarse a partir del nivel educativo de los padres y sus condiciones socioeconómicas, el carácter privado de la institución, la formación y el compromiso de los docentes de la institución y la existencia de personal de apoyo para el trabajo académico (psicóloga y psico-orientadora). De acuerdo con los planteamientos de Gaviria \& Barrientos (2001) los padres más educados poseen mayores recursos para proporcionar a sus hijos una mejor educación, tienden a dedicarles más tiempo a su instrucción y son más productivos en esta tarea.

Estudios han logrado establecer que los estudiantes de instituciones de carácter privado, tienen mejor rendimiento académico que los de 
instituciones oficiales. Los resultados de las pruebas ICFES para 1999 muestran que a nivel nacional, los puntajes de los estudiantes de colegios privados fueron 18.3 puntos más altos que los de sus pares en colegios públicos. Este resultado no es sorprendente si se considera que los estudiantes de colegios privados provienen de familias con mayores ingresos económicos, y consecuentemente tienen mejor nutrición, generalmente no tienen que trabajar, pertenecen a hogares más educados, tienen acceso a mejores útiles y ayudas escolares y asisten a colegios con mejores instalaciones (Núñez, Steiner, Cadena $\&$ Pardo, 2002). Por otra parte en lo relacionado con la formación docente podría afirmarse que la actualización, capacitación y formación del profesorado es importante para mejorar los logros de los estudiantes. En los últimos años se ha logrado demostrar la relación existente entre el desarrollo docente y el rendimiento escolar. Hay evidencia que muestra que incluso un breve curso de capacitación puede producir cambios en el comportamiento docente y en el rendimiento del alumno (Arancibia, 1994, citado en Torres, 1996). Así mismo el trabajo docente puede ser reforzado por personal de apoyo que sugiera nuevas estrategias para enfrentar las dificultades de los estudiantes. Los colegios de carácter privado cuentan generalmente con psicólogos y psicoorientadores, que apoyan el trabajo docente, y aunque no es muy claro el efecto que éstos puedan tener sobre el logro escolar, un mayor número de personal de apoyo puede ser un indicador de buena calidad (Núñez et al., 2002).

Rendimiento en Educación Media, de egresadas de El Colegio El Divino Niño, que cursan programas relacionados con Ciencias Naturales.

En el presente estudio se asumen como programas relacionados con Ciencias Naturales las carreras universitarias de Ingeniería, de Salud y Ciencias Básicas A continuación se presenta el porcentaje de estudiantes de la muestra que cursan programas relacionados con las Ciencias Naturales.

Tabla 8 .

Elección de programas relacionados con Ciencias

\begin{tabular}{|l|c|c|c|c|c|}
\hline \multicolumn{2}{|c|}{} & Frecuencia & Porcentaje & $\begin{array}{c}\text { Porcentaje } \\
\text { válido }\end{array}$ & $\begin{array}{c}\text { Porcentaje } \\
\text { acumulado }\end{array}$ \\
\hline \multirow{3}{*}{ Válidos } & $\mathrm{Si}$ & 31 & 29,2 & 29,2 & 29,2 \\
\cline { 2 - 6 } & No & 75 & 70,8 & 70,8 & 100,0 \\
\cline { 2 - 6 } & Total & 106 & 100,0 & 100,0 & \\
\hline
\end{tabular}

Los resultados de la tabla 8 indican que un $29.2 \%$ de las egresadas de El Colegio El Divino Niño cursan programas de pregrado relacionados con Ciencias Naturales y un $70,8 \%$ de ellas optan por otros programas. Teniendo en cuenta que el colegio de procedencia de las estudiantes ofrece una educación con profundización en esta área del conocimiento, se esperaría que el porcentaje de estudiantes que eligen carreras relacionadas con las ciencias fuera mayor, sin embargo si se compara este porcentaje con las estadísticas nacionales sobre demanda de los programas de pregrado, el porcentaje de egresadas que optaron por programas relacionados con las Ciencias Naturales puede considerarse alto. En general la baja demanda de los de los programas relacionados con las ciencias naturales (y en general de las Ciencias Básicas) podría explicarse atendiendo a que la escogencia del programa no depende solo de la formación recibida en el nivel precedente, sino también de otros factores externos como posibilidades y limitaciones de los estudiantes, entorno cultural, social y económico, la oferta educacional y la situación del mercado respecto a esa oferta. 
En este sentido, en la demanda de carreras científicas, especialmente en ciencias exactas como Biología, Química, Física y Matemáticas son varios los factores e instancias influyentes. En un estudio de la Universidad Nacional Autónoma de México, se señala como factor determinante en la baja demanda de estos programas a la también escasa demanda ocupacional que en muchos casos se traduce en subempleo (Domínguez \& Pérez, 1993). En concordancia con el anterior estudio, una investigación de la Universidad Javeriana (2002), afirma que la baja demanda en estos programas puede indicar la falta de incentivos para graduación de estudiantes, los cuales podrían provenir de las estructuras académicas mismas o del mercado en el que van a participar dichos graduados.

Desde otra perspectiva, puede señalarse en los últimos años, un pequeño incremento en la demanda de los programas relacionados con las Ciencias Básicas y Aplicadas. En una investigación adelantada en Argentina por Moler (2008), se logró determinar que analizando el total del sistema se registra un crecimiento del $2,4 \%$ sobre el total de las Ciencias Aplicadas y Básicas y un decrecimiento de menos del 1\% en las Ciencias Sociales Por otra parte, es indiscutible la influencia sobre la escogencia del programa a cursar de factores internos tales como las expectativas, motivaciones, necesidades, experiencias, aptitudes y valores. Con relación a este aspecto, entre las razones más influyentes en la escogencia del programa de pregrado, indicados por los inscritos al Examen del ICFES 2002-2003 se encuentran: las inclinaciones vocacionales, el deseo de servir a la comunidad y la posibilidad de alcanzar prestigio profesional, las cuales son razones relacionadas con las motivaciones personales del estudiante.

Tabla 9.

Rendimiento en Educación Media de egresadas que cursan programas relacionados con las Ciencias Naturales.

\begin{tabular}{|l|l|l|l|c|c|}
\hline \multicolumn{2}{|c|}{} & Frecuencia & Porcentaje & $\begin{array}{l}\text { Porcentaje } \\
\text { válido }\end{array}$ & $\begin{array}{c}\text { Porcentaje } \\
\text { acumulado }\end{array}$ \\
\hline \multirow{4}{*}{ Válidos } & Bajo & 1 & 3,2 & 3,2 & 3,2 \\
\cline { 2 - 6 } & Regular & 13 & 41,9 & 41,9 & 45,2 \\
\cline { 2 - 6 } & Alto & 17 & 54,8 & 54,8 & 100,0 \\
\cline { 2 - 6 } & Total & 31 & 100,0 & 100,0 & \\
\hline
\end{tabular}

Los resultados de la tabla 9 presentan que solo un $3.2 \%$ de las estudiantes que optaron por programas relacionados con las ciencias tienen rendimiento académico bajo en Educación Media y la gran mayoría de ellas presentan rendimiento académico satisfactorio en este nivel(un 54,8 con rendimiento alto), lo cual da cuenta del buen desempeño de las estudiantes durante la Educación Media.

¿Cuál es el rendimiento académico, de las egresadas que cursan programas de pregrado en la universidad?

Para describir el rendimiento académico en la universidad, se utilizará el concepto ya definido de Trayectoria Escolar, la cual se obtiene de la sumatoria de los niveles alcanzados en cada uno de los indicadores de rendimiento: (Ver Tabla 6). Consecuentemente para determinar la trayectoria escolar de cada estudiante es necesario establecer el nivel alcanzado en cada uno de los indicadores de rendimiento.

Trayectoria Escolar de las egresadas de El Colegio El Divino Niño

En la tabla 10 se observa que el $50.9 \%$ de las estudiantes evidencian una trayectoria escolar alta, un $34 \%$ una trayectoria regular y un $15.1 \%$ una trayectoria baja. Estos resultados indican un $84.9 \%$ de estudiantes con rendimiento académico 
satisfactorio, porcentaje que no registra mucha variación al compararlo con el $86.7 \%$ de estudiantes con rendimiento académico satisfactorio en Educación Media. Esto indica una tendencia a mejorar el rendimiento académico en la universidad. El porcentaje de estudiantes con trayectoria escolar baja aumentó en un 1.9\% con relación al porcentaje de estudiantes con rendimiento académico bajo en Educación Media, diferencia porcentual que no es muy significativa.

Estos resultados podrían explicarse a partir de la formación académica general recibida en el nivel precedente (Escudero 1981; citado en Musayón 2001), por la motivación de los estudiantes (García \& Doménech, 1997), e incluso por la formación y experiencia de los docentes de la universidad. Se ha logrado determinar que el conocimiento del tema por parte del maestro, su experiencia en el manejo de material didáctico y su expectativa con respecto al desempeño de los alumnos también están asociados con un incremento del logro académico de los estudiantes. También podrían considerarse como factores influyentes el nivel de exigencia de las universidades y de los programas académicos por los cuales optaron las estudiantes.

Son varios los estudios que dan cuenta de la relación entre el rendimiento académico en el nivel previo y el rendimiento académico futuro. Galaz et al. (s.f.), en su investigación sobre el éxito o fracaso académico de los estudiantes en la Universidad de Sonora, lograron establecer que el promedio obtenido en el bachillerato se relaciona de manera significativa con el promedio de la universidad. En este mismo sentido Tirado, Backhoff, Larrazolo \& Rojas (2005), concluyeron que los promedios escolares que tienen los alumnos pueden ser utilizados como un buen indicador de su ejecución futura, y por lo tanto deberían considerarse para pronosticar el desempeño escolar. Muchas otras investigaciones (García, Alvarado y Jiménez, 2000; Bastias, Villarroel, Zúñiga, Marshall, Velasco \& Mena, 2000; Valderrama, 2001; Martínez \& Solís, 2001; Edel, 2003a; Porto, Di Gresia y López, 2005; Duarte \& Galaz, 2006; Arias, Chávez y Muñoz, 2006; Cortés \& Palomar, 2008), lograron concluir que el mejor predictor del rendimiento académico futuro es el rendimiento anterior.

Por otra parte, si se analiza la influencia de los factores motivacionales en el rendimiento académico, no puede desconocerse que las metas que se trazan los estudiantes, dan lugar a diferentes modos de afrontar las tareas académicas y a distintos patrones motivacionales. Las motivaciones de algunos estudiantes pueden ser de carácter interno tales como el deseo de aprender, el retar sus propias capacidades e incluso la curiosidad, pero también existen motivaciones extrínsecas que pueden influir en el rendimiento como la obtención de notas, la aprobación de los padres, las recompensas y la obtención de juicios positivos. Estas dos orientaciones motivacionales están influenciadas por las creencias y convicciones del estudiante, las cuales le orientarán para elegir el modo de enfrentar la tarea o compromiso académico. Sin embargo, parece ser que las motivaciones intrínsecas tienen mayor influencia sobre el rendimiento de los alumnos.

Tabla 10.

Trayectoria Escolar de las egresadas de El Colegio El Divino Niño

\begin{tabular}{|l|l|l|c|c|c|}
\hline \multicolumn{2}{|c|}{} & Frecuencia & Porcentaje & $\begin{array}{l}\text { Porcentaje } \\
\text { válido }\end{array}$ & $\begin{array}{c}\text { Porcentaje } \\
\text { acumulado }\end{array}$ \\
\hline \multirow{3}{*}{ Válidos } & Baja & 16 & 15,1 & 15,1 & 15,1 \\
\cline { 2 - 6 } & Regular & 36 & 34,0 & 34,0 & 49,1 \\
\cline { 2 - 6 } & Alta & 54 & 50,9 & 50,9 & 100,0 \\
\cline { 2 - 6 } & Total & 106 & 100,0 & 100,0 & \\
\hline
\end{tabular}


Al observar la tabla 11 y 12 , se logra determinar que un importante porcentaje de estudiantes tiene una trayectoria escolar alta, sin embargo, al comparar el promedio y la trayectoria escolar, pueden observarse variaciones significativas que se explican por la influencia de los indicadores de rendimiento IAO e IP en la determinación del rendimiento académico en la universidad.

En la tabla 12 puede observarse que las estudiantes con promedio alto, obtienen trayectorias altas, pero también, estudiantes con promedios regulares pueden obtener trayectorias altas, regulares o bajas, aspecto en el cual se evidencia la influencia del IAO y del IP en la determinación de la trayectoria escolar. El porcentaje de estudiantes con promedios y trayectorias altas es de $27,35 \%$ con promedio regular y trayectoria alta es de $23.58 \%$, con promedio regular y trayectoria regular es de $33,96 \%$ y el de de promedios regulares y trayectorias bajas es de 3,77\%. En términos generales, la consideración del IAO y del IP (además del promedio), para determinar la trayectoria escolar de las estudiantes, permitió que un mayor porcentaje de las mismas se ubicara con rendimiento académico alto en la universidad.

Tabla 11.

Comparativo 1. Promedio y Trayectoria Escolar

\begin{tabular}{|l|c|c|}
\hline Clasificación & Promedio & Trayectoria \\
\hline Bajo (a) & $10,4 \%$ & $15,1 \%$ \\
\hline Regular & $61,3 \%$ & $34,0 \%$ \\
\hline Alto (a) & $28,3 \%$ & $50,9 \%$ \\
\hline
\end{tabular}

Tabla 12.

Comparativo 2. Promedio y Trayectoria Escolar

\begin{tabular}{|l|l|l|l|}
\hline & Trayectoria Alta & Trayectoria Regular & Trayectoria Baja \\
\hline Promedio Alto & $27.35 \%$ & $0 \%$ & $0 \%$ \\
\hline Promedio Regular & $23.58 \%$ & $33.96 \%$ & $3.77 \%$ \\
\hline Promedio Bajo & $0 \%$ & $0 \%$ & $11.32 \%$ \\
\hline
\end{tabular}

Rendimiento en la Universidad de egresadas que cursan programas relacionados con las Ciencias Naturales.

Los resultados de las tablas 13, 14, 15 y 16 dejan ver que de las estudiantes que optaron por programas relacionados con las ciencias naturales, un $77,4 \%$ presentan promedios regulares en la universidad. Así mismo la mayoría (71\%) tienen IAO altos. Con relación al IP puede afirmarse que no hay diferencia significativa entre los porcentajes de estudiantes con IP alto (45.2\%) y el porcentaje con IP regular (48.4\%). Al observar las trayectorias escolares se puede determinar que el mayor porcentaje de estudiantes presenta trayectoria escolar alta (45.2\%) seguido por un $38,7 \%$ con trayectoria regular, es decir, la mayoría de las estudiantes $(83.9 \%)$ presentan trayectoria escolar satisfactoria.

Tabla 13.

Promedio en la Universidad de las egresadas que cursan programas relacionados con las Ciencias Naturales

\begin{tabular}{|l|l|c|c|c|c|}
\hline \multicolumn{2}{|c|}{} & Frecuencia & Porcentaje & Porcentaje válido & Porcentaje acumulado \\
\hline Válidos & Bajo & 3 & 9,7 & 9,7 & 9,7 \\
\cline { 2 - 6 } & Regular & 24 & 77,4 & 77,4 & 87,1 \\
\cline { 2 - 6 } & Alto & 4 & 12,9 & 12,9 & 100,0 \\
\cline { 2 - 6 } & Total & 31 & 100,0 & 100,0 & \\
\hline
\end{tabular}


Tabla 14.

Índice de Aprobación en Ordinario

\begin{tabular}{|l|l|c|c|c|c|}
\hline \multicolumn{2}{|c|}{} & Frecuencia & Porcentaje & $\begin{array}{l}\text { Porcentaje } \\
\text { válido }\end{array}$ & $\begin{array}{c}\text { Porcentaje } \\
\text { acumulado }\end{array}$ \\
\hline \multirow{3}{*}{ Válidos } & Bajo & 3 & 9,7 & 9,7 & 9,7 \\
\cline { 2 - 6 } & Regular & 6 & 19,4 & 19,4 & 29,0 \\
\cline { 2 - 6 } & Alto & 22 & 71,0 & 71,0 & 100,0 \\
\cline { 2 - 6 } & Total & 31 & 100,0 & 100,0 & \\
\hline
\end{tabular}

Tabla 15.

Índice de promoción

\begin{tabular}{|l|c|c|c|c|c|}
\hline \multicolumn{2}{|c|}{} & Frecuencia & Porcentaje & $\begin{array}{l}\text { Porcentaje } \\
\text { válido }\end{array}$ & $\begin{array}{c}\text { Porcentaje } \\
\text { acumulado }\end{array}$ \\
\hline \multirow{3}{*}{ Válidos } & Bajo & 2 & 6,5 & 6,5 & 6,5 \\
\cline { 2 - 6 } & Regular & 15 & 48,4 & 48,4 & 54,8 \\
\cline { 2 - 6 } & Alto & 14 & 45,2 & 45,2 & 100,0 \\
\cline { 2 - 6 } & Total & 31 & 100,0 & 100,0 & \\
\hline
\end{tabular}

Tabla 16.

Trayectoria Escolar

\begin{tabular}{|l|l|c|c|c|c|}
\hline \multicolumn{2}{|c|}{} & Frecuencia & Porcentaje & $\begin{array}{l}\text { Porcentaje } \\
\text { válido }\end{array}$ & $\begin{array}{c}\text { Porcentaje } \\
\text { acumulado }\end{array}$ \\
\hline \multirow{3}{*}{ Válidos } & Baja & 5 & 16,1 & 16,1 & 16,1 \\
\cline { 2 - 6 } & Regular & 12 & 38,7 & 38,7 & 54,8 \\
\cline { 2 - 6 } & Alta & 14 & 45,2 & 45,2 & 100,0 \\
\cline { 2 - 6 } & Total & 31 & 100,0 & 100,0 & \\
\hline
\end{tabular}

De los resultados obtenidos, llama particularmente la atención que a pesar de que varios estudios muestran que existen altos niveles de reprobación en los programas de Ciencias Básicas (Noriega, 1989, Brito \& Amado, 2007), la mayoría de las egresadas de El Colegio El Divino Niño que cursan carreras universitarias relacionadas con las Ciencias Naturales, presentan promedios y trayectorias escolares satisfactorias (ver tablas 13 y 16).
¿En qué programas de Educación Superior evidencian mejor rendimiento académico las egresadas del Colegio El Divino Niño?

En la siguiente tabla se presentan los datos relacionados con la elección de programas y el rendimiento en Educación Media y en la universidad por parte de las egresadas de El Colegio El Divino Niño. 
Tabla 17.

Comparativo del rendimiento por Programa Académico.

\begin{tabular}{|c|c|c|c|c|c|c|}
\hline Programa & $\begin{array}{l}\text { Número de } \\
\text { Estudiantes }\end{array}$ & $\begin{array}{l}\text { Rendimiento } \\
\text { en Educación } \\
\text { Media }\end{array}$ & $\begin{array}{l}\text { Promedio } \\
\text { en la } \\
\text { Universidad }\end{array}$ & IAO & IP & Trayectoria \\
\hline \multirow{3}{*}{$\begin{array}{l}\text { Negocios } \\
\text { Internacionales }\end{array}$} & & $\mathrm{A}=28.6 \%$ & $A=42.9 \%$ & $\mathrm{~A}=85.7 \%$ & $\mathrm{~A}=100 \%$ & $\mathrm{~A}=85.7 \%$ \\
\hline & 7 & $\mathrm{R}=57.1 \%$ & $\mathrm{R}=57.1 \%$ & $\mathrm{R}=14.3 \%$ & $\mathrm{R}=0 \%$ & $\mathrm{R}=14.3 \%$ \\
\hline & & $\mathrm{B}=14.3 \%$ & $\mathrm{~B}=0 \%$ & $\mathrm{~B}=0 \%$ & $\mathrm{~B}=0 \%$ & $\mathrm{~B}=0 \%$ \\
\hline \multirow{3}{*}{$\begin{array}{l}\text { Ingeniería } \\
\text { Industrial }\end{array}$} & & $\mathrm{A}=62.5 \%$ & $\mathrm{~A}=12.5 \%$ & $A=75 \%$ & $\mathrm{~A}=50 \%$ & $\mathrm{~A}=37.5 \%$ \\
\hline & 7 & $\mathrm{R}=37.5 \%$ & $\mathrm{R}=87.5 \%$ & $\mathrm{R}=25 \%$ & $\mathrm{R}=50 \%$ & $\mathrm{R}=62.5 \%$ \\
\hline & & $\mathrm{B}=0 \%$ & $\mathrm{~B}=0 \%$ & $\mathrm{~B}=0 \%$ & $\mathrm{~B}=0 \%$ & $\mathrm{~B}=0 \%$ \\
\hline \multirow{3}{*}{$\begin{array}{l}\text { Administración } \\
\text { de Empresas }\end{array}$} & & $A=10 \%$ & $A=10 \%$ & $\mathrm{~A}=30 \%$ & $A=50 \%$ & $\mathrm{~A}=30 \%$ \\
\hline & 10 & $\mathrm{R}=90 \%$ & $\mathrm{R}=70 \%$ & $\mathrm{R}=60 \%$ & $\mathrm{R}=50 \%$ & $\mathrm{R}=50 \%$ \\
\hline & & $\mathrm{B}=0 \%$ & $\mathrm{~B}=20 \%$ & $\mathrm{~B}=10 \%$ & $\mathrm{~B}=0 \%$ & $\mathrm{~B}=20 \%$ \\
\hline \multirow{3}{*}{$\begin{array}{l}\text { Ingeniería } \\
\text { Ambiental }\end{array}$} & & $\mathrm{A}=33.3 \%$ & $\mathrm{~A}=22.2 \%$ & $A=55.6 \%$ & $\mathrm{~A}=11.1 \%$ & $\mathrm{~A}=22.2 \%$ \\
\hline & 9 & $\mathrm{R}=55.6 \%$ & $\mathrm{R}=44.4 \%$ & $\mathrm{R}=33.3 \%$ & $\mathrm{R}=77.8 \%$ & $\mathrm{R}=44.4 \%$ \\
\hline & & $\mathrm{B}=11.1 \%$ & $\mathrm{~B}=33.3 \%$ & $\mathrm{~B}=11.1 \%$ & $\mathrm{~B}=11.1 \%$ & $\mathrm{~B}=33.3 \%$ \\
\hline \multirow[t]{3}{*}{ Psicología } & & $\mathrm{A}=0 \%$ & $\mathrm{~A}=0 \%$ & $\mathrm{~A}=83.3 \%$ & $\mathrm{~A}=33.3 \%$ & $\mathrm{~A}=16.7 \%$ \\
\hline & 6 & $\mathrm{R}=66.7 \%$ & $\mathrm{R}=100 \%$ & $\mathrm{R}=16.7 \%$ & $\mathrm{R}=66.7 \%$ & $\mathrm{R}=83.3 \%$ \\
\hline & & $\mathrm{B}=33.3 \%$ & $\mathrm{~B}=0 \%$ & $\mathrm{~B}=0 \%$ & $\mathrm{~B}=0 \%$ & $\mathrm{~B}=0 \%$ \\
\hline \multirow{3}{*}{ Contaduría } & & $\mathrm{A}=0 \%$ & $\mathrm{~A}=0 \%$ & $\mathrm{~A}=0 \%$ & $\mathrm{~A}=100 \%$ & $\mathrm{~A}=0 \%$ \\
\hline & 1 & $\mathrm{R}=100 \%$ & $\mathrm{R}=100 \%$ & $\mathrm{R}=100 \%$ & $\mathrm{R}=0 \%$ & $\mathrm{R}=100 \%$ \\
\hline & & $\mathrm{B}=0 \%$ & $\mathrm{~B}=0 \%$ & $\mathrm{~B}=0 \%$ & $\mathrm{~B}=0 \%$ & $\mathrm{~B}=0 \%$ \\
\hline \multirow[t]{3}{*}{ Medicina } & & $\mathrm{A}=50 \%$ & $\mathrm{~A}=0 \%$ & $\mathrm{~A}=83.3 \%$ & $\mathrm{~A}=83.3 \%$ & $\mathrm{~A}=66.7 \%$ \\
\hline & 6 & $\mathrm{R}=50 \%$ & $\mathrm{R}=83.3 \%$ & $\mathrm{R}=0 \%$ & $\mathrm{R}=16.7 \%$ & $\mathrm{R}=16.7 \%$ \\
\hline & & $\mathrm{B}=0 \%$ & $\mathrm{~B}=16.7 \%$ & $\mathrm{~B}=16.7 \%$ & $\mathrm{~B}=0 \%$ & $\mathrm{~B}=16.7 \%$ \\
\hline \multirow{3}{*}{$\begin{array}{l}\text { Cine y } \\
\text { Audiovisuales }\end{array}$} & & $\mathrm{A}=0 \%$ & $A=50 \%$ & $A=100 \%$ & $\mathrm{~A}=50 \%$ & $\mathrm{~A}=50 \%$ \\
\hline & 2 & $\mathrm{R}=50 \%$ & $\mathrm{R}=50 \%$ & $\mathrm{R}=0 \%$ & $\mathrm{R}=50 \%$ & $\mathrm{R}=50 \%$ \\
\hline & & $\mathrm{B}=50 \%$ & $\mathrm{~B}=0 \%$ & $\mathrm{~B}=0 \%$ & $\mathrm{~B}=0 \%$ & $\mathrm{~B}=0 \%$ \\
\hline \multirow[t]{3}{*}{ Antropología } & & $\mathrm{A}=0 \%$ & $\mathrm{~A}=0 \%$ & $\mathrm{~A}=50 \%$ & $\mathrm{~A}=0 \%$ & $\mathrm{~A}=0 \%$ \\
\hline & 2 & $\mathrm{R}=100 \%$ & $\mathrm{R}=100 \%$ & $\mathrm{R}=50 \%$ & $\mathrm{R}=100 \%$ & $\mathrm{R}=100 \%$ \\
\hline & & $\mathrm{B}=0 \%$ & $\mathrm{~B}=0 \%$ & $\mathrm{~B}=0 \%$ & $\mathrm{~B}=0 \%$ & $\mathrm{~B}=0 \%$ \\
\hline \multirow[t]{3}{*}{ Odontología } & & $\mathrm{A}=0 \%$ & $\mathrm{~A}=0 \%$ & $\mathrm{~A}=50 \%$ & $\mathrm{~A}=50 \%$ & $\mathrm{~A}=50 \%$ \\
\hline & 2 & $\mathrm{R}=100 \%$ & $\mathrm{R}=100 \%$ & $\mathrm{R}=50 \%$ & $\mathrm{R}=50 \%$ & $\mathrm{R}=50 \%$ \\
\hline & & $\mathrm{B}=0 \%$ & $\mathrm{~B}=0 \%$ & $\mathrm{~B}=0 \%$ & $\mathrm{~B}=0 \%$ & $\mathrm{~B}=0 \%$ \\
\hline \multirow[t]{3}{*}{ Biología } & & $A=50 \%$ & $\mathrm{~A}=0 \%$ & $A=50 \%$ & $\mathrm{~A}=0 \%$ & $A=0 \%$ \\
\hline & 2 & $\mathrm{R}=50 \%$ & $\mathrm{R}=50 \%$ & $\mathrm{R}=0 \%$ & $\mathrm{R}=50 \%$ & $\mathrm{R}=50 \%$ \\
\hline & & $\mathrm{B}=0 \%$ & $\mathrm{~B}=50 \%$ & $\mathrm{~B}=50 \%$ & $\mathrm{~B}=50 \%$ & $\mathrm{~B}=50 \%$ \\
\hline \multirow[t]{3}{*}{ Derecho } & & $\mathrm{A}=31.6 \%$ & $\mathrm{~A}=77.8 \%$ & $\mathrm{~A}=78.9 \%$ & $\mathrm{~A}=57.9 \%$ & $\mathrm{~A}=68.4 \%$ \\
\hline & 19 & $\mathrm{R}=52.6 \%$ & $\mathrm{R}=22.2 \%$ & $\mathrm{R}=10.5 \%$ & $\mathrm{R}=31.6 \%$ & $\mathrm{R}=10.5 \%$ \\
\hline & & $\mathrm{B}=15.8 \%$ & $\mathrm{~B}=0 \%$ & $\mathrm{~B}=10.5 \%$ & $\mathrm{~B}=10.5 \%$ & $\mathrm{~B}=21.1 \%$ \\
\hline \multirow{3}{*}{$\begin{array}{l}\text { Comunicación } \\
\text { Social }\end{array}$} & & $\mathrm{A}=11.1 \%$ & $A=55.6 \%$ & $\mathrm{~A}=100 \%$ & $\mathrm{~A}=77.8 \%$ & $\mathrm{~A}=77.8 \%$ \\
\hline & 9 & $\mathrm{R}=66.7 \%$ & $\mathrm{R}=44.4 \%$ & $\mathrm{R}=0 \%$ & $\mathrm{R}=22.2 \%$ & $\mathrm{R}=22.2 \%$ \\
\hline & & $\mathrm{B}=22.2 \%$ & $\mathrm{~B}=0 \%$ & $\mathrm{~B}=0 \%$ & $\mathrm{~B}=0 \%$ & $\mathrm{~B}=0 \%$ \\
\hline \multirow{3}{*}{ Finanzas } & & $\mathrm{A}=28.6 \%$ & $\mathrm{~A}=50 \%$ & $\mathrm{~A}=78.6 \%$ & $\mathrm{~A}=64.3 \%$ & $\mathrm{~A}=64.3 \%$ \\
\hline & 14 & $\mathrm{R}=71.4 \%$ & $\mathrm{R}=50 \%$ & $\mathrm{R}=21.4 \%$ & $\mathrm{R}=21.4 \%$ & $\mathrm{R}=21.4 \%$ \\
\hline & & $\mathrm{B}=0 \%$ & $\mathrm{~B}=0 \%$ & $\mathrm{~B}=0 \%$ & $\mathrm{~B}=14.3 \%$ & $\mathrm{~B}=14.3 \%$ \\
\hline \multirow{3}{*}{$\begin{array}{l}\text { Marketing y } \\
\text { Negocios } \\
\text { Internacionales }\end{array}$} & & $\mathrm{A}=0 \%$ & $\mathrm{~A}=0 \%$ & $\mathrm{~A}=25 \%$ & $\mathrm{~A}=0 \%$ & $\mathrm{~A}=0 \%$ \\
\hline & 4 & $\mathrm{R}=0 \%$ & $\mathrm{R}=25 \%$ & $\mathrm{R}=25 \%$ & $\mathrm{R}=25 \%$ & $\mathrm{R}=25 \%$ \\
\hline & & $\mathrm{B}=100 \%$ & $B=75 \%$ & $\mathrm{~B}=50 \%$ & $\mathrm{~B}=75 \%$ & $\mathrm{~B}=75 \%$ \\
\hline Microbiología & 1 & $\mathrm{~A}=100 \%$ & $\mathrm{~A}=100 \%$ & $\mathrm{~A}=100 \%$ & $\mathrm{~A}=100 \%$ & $\mathrm{~A}=100 \%$ \\
\hline & & $\mathrm{R}=0 \%$ & $\mathrm{R}=0 \%$ & $\mathrm{R}=0 \%$ & $\mathrm{R}=0 \%$ & $\mathrm{R}=0 \%$ \\
\hline & & $\mathrm{B}=0 \%$ & $\mathrm{~B}=0 \%$ & $\mathrm{~B}=0 \%$ & $\mathrm{~B}=0 \%$ & $\mathrm{~B}=0 \%$ \\
\hline Ingeniería & & $\mathrm{A}=100 \%$ & $\mathrm{~A}=0 \%$ & $\mathrm{~A}=0 \%$ & $\mathrm{~A}=0 \%$ & $\mathrm{~A}=0 \%$ \\
\hline Electrónica & 1 & $\mathrm{R}=0 \%$ & $\mathrm{R}=100 \%$ & $\mathrm{R}=100 \%$ & $\mathrm{R}=100 \%$ & $\mathrm{R}=100 \%$ \\
\hline & & $\mathrm{B}=0 \%$ & $\mathrm{~B}=0 \%$ & $\mathrm{~B}=0 \%$ & $\mathrm{~B}=0 \%$ & $\mathrm{~B}=0 \%$ \\
\hline Ingeniería de & & $\mathrm{A}=100 \%$ & $\mathrm{~A}=0 \%$ & $\mathrm{~A}=100 \%$ & $A=100 \%$ & $A=100 \%$ \\
\hline Energía & 1 & $\mathrm{R}=0 \%$ & $\mathrm{R}=100 \%$ & $\mathrm{R}=0 \%$ & $\mathrm{R}=0 \%$ & $\mathrm{R}=0 \%$ \\
\hline & & $\mathrm{B}=0 \%$ & $\mathrm{~B}=0 \%$ & $\mathrm{~B}=0 \%$ & $\mathrm{~B}=0 \%$ & $\mathrm{~B}=0 \%$ \\
\hline Ingeniería & & $A=100 \%$ & $\mathrm{~A}=0 \%$ & $\mathrm{~A}=100 \%$ & $\mathrm{~A}=100 \%$ & $A=100 \%$ \\
\hline Civil & 1 & $\mathrm{R}=0 \%$ & $\mathrm{R}=100 \%$ & $\mathrm{R}=0 \%$ & $\mathrm{R}=0 \%$ & $\mathrm{R}=0 \%$ \\
\hline & & $\mathrm{B}=0 \%$ & $\mathrm{~B}=0 \%$ & $\mathrm{~B}=0 \%$ & $\mathrm{~B}=0 \%$ & $\mathrm{~B}=0 \%$ \\
\hline Ingeniería de & & $\mathrm{A}=100 \%$ & $\mathrm{~A}=0 \%$ & $\mathrm{~A}=100 \%$ & $\mathrm{~A}=100 \%$ & $\mathrm{~A}=100 \%$ \\
\hline Petróleos & 1 & $\mathrm{R}=0 \%$ & $\mathrm{R}=100 \%$ & $\mathrm{R}=0 \%$ & $\mathrm{R}=0 \%$ & $\mathrm{R}=0 \%$ \\
\hline & & $\mathrm{B}=0 \%$ & $\mathrm{~B}=0 \%$ & $\mathrm{~B}=0 \%$ & $\mathrm{~B}=0 \%$ & $\mathrm{~B}=0 \%$ \\
\hline
\end{tabular}


Puede establecerse que el programa de pregrado con mayor demanda es el de derecho seguido por finanzas y administración ya que el 40.5\% ( 43 estudiantes) de la muestra cursan estos programas. Un $29.2 \%$ (31 estudiantes) optan por programas relacionados con las ciencias naturales (Ingenierías, Odontología, Medicina, Biología y Microbiología) y el 30.3\% restante (32 estudiantes) optan por otros programas. En este sentido, Viloria (2006) en su estudio sobre "Educación Superior en el Caribe Colombiano" manifiesta que los programas de ciencias económicas tienen la mayor demanda, seguidos por ingenierías, arquitectura, urbanismo y similares. En estas áreas se concentra el $50 \%$ del total de la matrícula. Por el contrario, los programas de matemáticas, ciencias naturales, agronomía, veterinaria, humanidades y ciencias religiosas sólo concentran el $4 \%$ de la matrícula.

Por otra parte, un informe elaborado por el Ministerio de Educación Nacional (2003), registra que las carreras universitarias de mayor preferencia siguen siendo aquellas que tradicionalmente han estado asociadas al prestigio social y económico, tales como la medicina, ingenierías, enfermería, psicología, derecho, administración y odontología, según la información registrada por los inscritos en el Examen de Estado 2002-2003. Al comparar los anteriores resultados, con los pregrados preferidos por los estudiantes encuestados en el estudio realizado por Universidad Nacional, Sede Medellín, la tendencia se mantiene debido a que existe una marcada preferencia hacia los pregrados relacionados con las ciencias de la salud y en su orden le siguen administración de negocios, comunicación social, ingenierías y derecho. (Universidad Nacional, Sede Medellín; 2006).

Las ingenierías, constituyen los programas de mayor demanda por parte del grupo de estudiantes que cursan estudios universitarios relacionados con las ciencias ya que 20, de ellas están matriculadas en estos programas Un estudio adelantado por la facultad de Ciencias Económicas de la Universidad Javeriana (2002) logró establecer que las carreras que presentaban mayor demanda en Colombia para el año 1999 eran Ciencias Económicas e Ingenierías, representando entre los dos grupos más del $55 \%$ del total de alumnos matriculados. Le seguían las carreras de Ciencias Sociales y Ciencias de la Educación con alrededor de 15\% de la matrícula total cada una. Seguían las carreras en Ciencias de la Salud y Ciencias Naturales con $11 \%$ del total de matrícula. Finalmente, el último $4 \%$ se repartía entre las carreras de Bellas Artes, Agronomía y Veterinaria y Humanidades. Además al estimar la tasa de crecimiento se muestra que en general, las carreras de mayor demanda (Ciencias Económicas y Administrativas y las de Ingeniería) son también carreras con altas tasas de crecimiento cuantitativo. En este mismo sentido, Viloria afirma que la preferencia por los estudios en alguna especialización en ingeniería se ha incrementado en más de diez puntos porcentuales en la Costa Caribe, comparándolos con el año 1990 (Viloria, 2006).

De acuerdo con los resultados de la tabla 17 las estudiantes que eligieron programas de Ingenierías, Microbiología, Biología y Medicina presentaron mejor rendimiento en Educación Media que el resto de sus compañeras. Podría afirmarse que las estudiantes que optaron por programas relacionados con las Ciencias Naturales, obtuvieron rendimiento alto en Educación Media. Por su parte ninguna de las estudiantes que optaron por los programas de Psicología, Contaduría, Cine y Audiovisuales, Antropología y Marketing obtuvo rendimiento académico alto en Educación Media y de las que eligieron los programas de Derecho, Administración de Empresas, Comunicación Social y Finanzas presentaron en su mayoría rendimiento académico regular en este nivel educativo.

Los resultados también indican que las estudiantes que cursan programas de Ingenierías (Petróleos, Energía y Civil), Medicina y 
Microbiología, mantienen alto rendimiento académico en la universidad, mientras que aquellas que estudian Ingeniería Ambiental, Ingeniería Civil, Ingeniería Electrónica y Biología disminuyeron su rendimiento académico en la universidad con relación a su rendimiento en Educación Media.

Además, al analizar las trayectorias escolares, se puede determinar que las estudiantes que optaron por los programas de Negocios Internacionales, Comunicación Social, Derecho, y Finanzas, presentan también trayectoria escolar alta aunque el mayor porcentaje de estas, presentaron rendimiento académico regular o bajo en Educación Media

\section{¿Existe relación entre el rendimiento académico de las egresadas en su Educación Media y su rendimiento en la universidad?}

Correlación entre Rendimiento en Educación Media y Trayectoria Escolar.

El valor de la correlación ñ para las variables rendimiento en Educación Media y trayectoria escolar, indicada en la tabla 18, es significativa y muestra una moderada asociación entre estas variables con un valor de 0,417 al nivel de significancia 0,01 . En los estudios predictivos es razonable esperar correlaciones modestas que van de 0,3 a 0,4 (Nunnally \& Bernstein, 1981 citado en Arias et al., 2006).

Tabla 18.

Relación Rendimiento en Educación Media y Trayectoria

\begin{tabular}{|c|c|c|c|c|}
\hline & & & $\begin{array}{l}\text { Rendimiento } \\
\text { en Educación Media }\end{array}$ & Trayectoria Escolar \\
\hline \multirow[t]{6}{*}{ Rho de Spearman } & \multirow[t]{3}{*}{$\begin{array}{l}\text { Rendimiento en } \\
\text { Educación Media }\end{array}$} & $\begin{array}{l}\text { Coeficiente } \\
\text { de correlación }\end{array}$ & 1,000 &, $417(* *)$ \\
\hline & & Sig. (bilateral) & & ,000 \\
\hline & & $\mathrm{N}$ & 106 & 106 \\
\hline & \multirow[t]{3}{*}{$\begin{array}{l}\text { Trayectoria } \\
\text { Escolar }\end{array}$} & $\begin{array}{l}\text { Coeficiente de } \\
\text { correlación }\end{array}$ &, $417(* *)$ & 1,000 \\
\hline & & Sig. (bilateral) &, 000 & \\
\hline & & $\mathrm{N}$ & 106 & 106 \\
\hline
\end{tabular}

** La correlación es significativa al nivel 0,01 (bilateral).

La moderada asociación entre las variables rendimiento académico en Educación Media y trayectoria escolar es fácilmente observable al analizar los resultados de la tabla 18 En este caso particular estudiantes que presentaron rendimiento académico alto en Educación Media, evidencian en la universidad trayectorias escolares altas, regulares o bajas, estudiantes con rendimiento académico regular en Educación Media registran rendimiento académico alto regular o bajo en la Universidad y finalmente estudiantes con rendimiento académico bajo en Educación Media obtuvieron en la universidad rendimiento académico bajo, regular e inclusive alto.
Varios estudios, han permitido establecer que el rendimiento académico en la universidad está influenciado por varios factores entre ellos el rendimiento académico previo. Si bien es cierto no podría afirmarse que el rendimiento académico en el nivel precedente, tiene un valor predictivo, si puede establecerse que hay una tendencia a que las estudiantes mantengan en la universidad el rendimiento académico obtenido en Educación Media. En este sentido, los resultados de la investigación adelantada por Duarte, Galaz \& Rosales (2005) sugieren que un estudiante con buen rendimiento en el bachillerato cuenta con las características necesarias (hábitos y estrategias de estudio, buena actitud hacia el aprendizaje y 
condiciones socioeconómicas) para desempeñarse académicamente con un nivel alto en sus estudios universitarios. García et al. (2000), lograron establecer que el mejor predictor del rendimiento académico futuro es el rendimiento anterior. $\mathrm{Y}$ en este mismo sentido muchas otras investigaciones (Tirado et al., 1997, Bastias et al., 2000, Valderrama, 2001, Edel, 2003b, Arias et al., 2006, Cortés \& Palomar, 2008) concluyeron que uno de los principales indicadores del éxito académico de los alumnos se relaciona con las calificaciones obtenidas en sus estudios previos.

Sin embargo, los estudios sobre rendimiento académico también indican que son muchos los factores asociados con el mismo, razón por la cual la variación en el rendimiento académico en la universidad con relación al obtenido en Educación Media, puede atribuirse a muchos de ellos como los factores psicosociales(Morales \& Arcos, 1999; Andino, Cardozo, Duré, Falcon, Pierlorenzi, Gauna et al., 2003; Rojas, 2005), los factores emocionales y el autoconcepto (Herrera, 2004; Reyes, 2003), los estilos de aprendizaje (Dunn \& Dunn, 1979, citados en Cabrera \& Fariñas, s.f.; Cantú, 2004; Figueroa, Cataldi, Méndez, Rendón, Costa, Salgueiro et al., 2005), enfoques de aprendizaje (Valle, González, Núñez, Suárez, Piñeiro \& Rodríguez, 2000), la motivación (García \& Doménech, 1997, Andino et al., 2003) y el interés vocacional (Vélez \& Roa, 2005).

Podríamos seguir mencionando una gran variedad de factores que se asocian al rendimiento académico y que han sido y continuarán siendo objeto de muchas investigaciones. Es indiscutible entonces, que el rendimiento académico es un fenómeno complejo y multifactorial, por lo tanto atribuirle la influencia sobre el mismo a un o unos pocos factores sería arbitrario.

\section{¿Existe relación entre el rendimiento académico en ciencias naturales y matemáticas en Educación Media y el rendimiento en la universidad en las asignaturas relacionadas con estas áreas?}

Resultados relacionados con Rendimiento en Ciencias Naturales y Matemáticas.

Los resultados de las tablas 19 y 21 indican que un alto porcentaje de estudiantes evidenciaron un rendimiento académico regular en las áreas de Ciencias Naturales y Matemáticas (61,3\% y $67,7 \%$ respectivamente) y no se registran estudiantes con rendimiento académico bajo en éstas áreas durante su Educación Media. Sin embargo, al observarse los resultados de las tablas 20 y 22 , relacionados con los promedios académicos en la universidad, puede establecerse que la mayoría de las estudiantes $(45,2 \%)$ presentan promedios académicos regulares para Ciencias Naturales, seguido de un 32,3\% con promedios bajos. Para el área de Matemáticas se establece que un alto número de estudiantes $(45,2 \%)$ presentaron promedios bajos y un $38,7 \%$ evidenció promedios regulares. Así mismo el porcentaje de estudiantes con rendimiento alto en Educación Media disminuyó significativamente en la universidad, pasando de un 38,7\% a $22,6 \%$ en ciencias naturales y de un $32,3 \%$ a $12,9 \%$ en matemáticas.

Rendimiento Académico en Ciencias Naturales en Educación Media.

Tabla 19.

Rendimiento en Ciencias Naturales en Educación Media

\begin{tabular}{|l|l|c|c|c|c|}
\hline \multicolumn{2}{|c|}{} & Frecuencia & Porcentaje & $\begin{array}{l}\text { Porcentaje } \\
\text { válido }\end{array}$ & $\begin{array}{c}\text { Porcentaje } \\
\text { acumulado }\end{array}$ \\
\hline \multirow{3}{*}{ Válidos } & Regular & 19 & 61,3 & 61,3 & 61,3 \\
\cline { 2 - 6 } & Alto & 12 & 38,7 & 38,7 & 100,0 \\
\cline { 2 - 6 } & Total & 31 & 100,0 & 100,0 & \\
\hline
\end{tabular}


Promedio en Ciencias Naturales en la Universidad

Tabla 20.

Promedio en Ciencias Naturales en la Universidad

\begin{tabular}{|l|l|l|l|l|c|}
\hline \multicolumn{2}{|c|}{} & Frecuencia & Porcentaje & $\begin{array}{c}\text { Porcentaje } \\
\text { válido }\end{array}$ & $\begin{array}{c}\text { Porcentaje } \\
\text { acumulado }\end{array}$ \\
\hline Válidos & Bajo & 10 & 32,3 & 32,3 & 32,3 \\
\cline { 2 - 6 } & Regular & 14 & 45,2 & 45,2 & 77,4 \\
\cline { 2 - 6 } & Alto & 7 & 22,6 & 22,6 & 100,0 \\
\cline { 2 - 6 } & Total & 31 & 100,0 & 100,0 & \\
\hline
\end{tabular}

Rendimiento Académico en Matemáticas en Educación Media

Tabla 21.

Rendimiento en Matemáticas en Educación Media

\begin{tabular}{|l|l|l|c|c|c|}
\hline \multicolumn{2}{|c|}{} & Frecuencia & Porcentaje & $\begin{array}{l}\text { Porcentaje } \\
\text { válido }\end{array}$ & $\begin{array}{c}\text { Porcentaje } \\
\text { acumulado }\end{array}$ \\
\hline Válidos & Regular & 21 & 67,7 & 67,7 & 67,7 \\
\cline { 2 - 6 } & Alto & 10 & 32,3 & 32,3 & 100,0 \\
\cline { 2 - 6 } & Total & 31 & 100,0 & 100,0 & \\
\hline
\end{tabular}

Promedio en Matemáticas en la Universidad

Tabla 22.

Promedio en Matemáticas en la Universidad

\begin{tabular}{|l|l|c|c|c|c|}
\hline \multicolumn{2}{|c|}{} & Frecuencia & Porcentaje & $\begin{array}{c}\text { Porcentaje } \\
\text { válido }\end{array}$ & $\begin{array}{c}\text { Porcentaje } \\
\text { acumulado }\end{array}$ \\
\hline \multirow{3}{*}{ Válidos } & Bajo & 14 & 45,2 & 46,7 & 46,7 \\
\cline { 2 - 6 } & Regular & 12 & 38,7 & 40,0 & 86,7 \\
\cline { 2 - 6 } & Alto & 4 & 12,9 & 13,3 & 100,0 \\
\cline { 2 - 6 } & Total & 30 & 96,8 & 100,0 & \\
\hline
\end{tabular}

Estos resultados podrían atribuirse a la insuficiente preparación de las estudiantes durante la Educación Media en las áreas de matemáticas y ciencias naturales, a las divergentes metodologías utilizadas en los dos niveles (Educación Media y Educación Superior) e inclusive a los hábitos de estudio y al tiempo de dedicación al mismo. En este sentido afirman Addine \& Ramírez (2004) que la actual enseñanza de las ciencias va dirigida a preparar estudiantes aptos para enfrentar las carreras universitarias; pero no realmente a brindar una sólida cultura científica, por lo cual solo meses después de culminar el bachillerato muchos estudiantes han olvidado gran parte de lo que creían haber aprendido sobre ciencias y en ocasiones su conducta estará relacionada con ese importante déficit, es decir, esto se traducirá en bajos resultados en las asignaturas con alto contenido científico. Barrantes \& Sequeira (1993) lograron concluir que los resultados obtenidos con relación al tiempo utilizado por los estudiantes 
para estudiar matemáticas fuera de clase, reflejan que éstos carecen de buenos hábitos de estudio diario ya que acostumbran estudiar solamente para la prueba, pretendiendo abarcar en un corto tiempo toda la materia cubierta por el profesor.

Noriega (1989) en su investigación sobre el rendimiento escolar en Ciencias Básicas, realizada en el Instituto Tecnológico de Querétaro (México) logró establecer que una consecuencia inmediata de la condición deficiente que presenta el estudiante de nuevo ingreso, se traduce en elevados índices de reprobación especialmente en las materias de matemáticas, estática, dinámica, electricidad, magnetismo y química; así mismo identificó que las causas de estos altos índices de reprobación, se relacionan tanto con el estudiante como con el docente.

Fernández (s.f., citado en Robles, 2005) señala que en los últimos años los resultados obtenidos tanto en la Organización para la Cooperación y Desarrollo Económicos (OCDE) como en la UNESCO en términos del rendimiento académico son desfavorables, principalmente en matemáticas y ciencias naturales, y que sólo un porcentaje mínimo de la población escolar estudia carreras científicas y de las ciencias exactas. En este mismo sentido en un estudio de la Universidad Nacional Autónoma de México, se señala a la preparación de las escuelas de bachillerato como factor que redunda negativamente el nivel académico de las asignaturas de los primeros semestres relacionados con las ciencias (Domínguez \& Pérez, 1993).

Correlación entre Rendimiento Académico en Ciencias Naturales y Matemáticas en Educación Media y Promedio en asignaturas relacionadas con estas áreas en la Universidad.

Los resultados de las tablas 23 y 24 muestran una correlación de 0,203 y 0.249 , entre el rendimiento en Educación Media y promedio en la universidad para ciencias naturales y matemáticas respectivamente. Estas correlaciones pueden asumirse como muy débiles y no significativas, y explicarse a partir del bajo porcentaje de estudiantes que mantienen su rendimiento académico en la universidad en ciencias naturales y matemáticas, con relación al rendimiento obtenido en Educación Media en estas mismas áreas.

Tabla 23.

Relación Rendimiento en Ciencias Naturales en Educación Media y Promedio en Ciencias Naturales en la Universidad

\begin{tabular}{|c|c|c|c|c|}
\hline & & & $\begin{array}{l}\text { Rendimiento } \\
\text { en Ciencias } \\
\text { en Educ. Media }\end{array}$ & $\begin{array}{l}\text { Promedio en } \\
\text { Ciencias } \\
\text { Universidad }\end{array}$ \\
\hline \multirow[t]{6}{*}{ Rho de Spearman } & \multirow{3}{*}{$\begin{array}{l}\text { Rendimiento } \\
\text { en Ciencias en Educ. } \\
\text { Media }\end{array}$} & $\begin{array}{l}\text { Coeficiente de } \\
\text { correlación }\end{array}$ & 1,000 & ,203 \\
\hline & & Sig. (bilateral) & & ,273 \\
\hline & & $\mathrm{N}$ & 31 & 31 \\
\hline & \multirow{3}{*}{$\begin{array}{l}\text { Promedio en } \\
\text { Ciencias } \\
\text { Universidad }\end{array}$} & $\begin{array}{l}\text { Coeficiente de } \\
\text { correlación }\end{array}$ & ,203 & 1,000 \\
\hline & & Sig. (bilateral) & ,273 & \\
\hline & & $\mathrm{N}$ & 31 & 31 \\
\hline
\end{tabular}


Tabla 24.

Relación Rendimiento Académico en Matemáticas en Educación Media y Promedio en Matemáticas en la Universidad.

\begin{tabular}{|c|c|c|c|c|}
\hline & & & $\begin{array}{l}\text { Rendimiento } \\
\text { en Matemáticas } \\
\text { en Educ. Media }\end{array}$ & $\begin{array}{l}\text { Promedio en } \\
\text { Matemáticas } \\
\text { Universidad }\end{array}$ \\
\hline \multirow[t]{6}{*}{ Rho de Spearman } & \multirow{3}{*}{$\begin{array}{l}\text { Rendimiento en } \\
\text { Matemáticas } \\
\text { en Educ. Media }\end{array}$} & $\begin{array}{l}\text { Coeficiente de } \\
\text { correlación }\end{array}$ & 1,000 & ,249 \\
\hline & & Sig. (bilateral) & & ,185 \\
\hline & & $\mathrm{N}$ & 31 & 30 \\
\hline & \multirow{3}{*}{$\begin{array}{l}\text { Promedio en } \\
\text { Matemáticas } \\
\text { Universidad }\end{array}$} & $\begin{array}{l}\text { Coeficiente de } \\
\text { correlación }\end{array}$ & ,249 & 1,000 \\
\hline & & Sig. (bilateral) & ,185 & \\
\hline & & $\mathrm{N}$ & 30 & 30 \\
\hline
\end{tabular}

En general, las estudiantes que cursan programas relacionadas con las Ciencias Naturales presentan trayectorias escolares satisfactorias $(83,9 \%)$, sin embargo al analizar aisladamente el rendimiento académico en las asignaturas básicas del programa, éste desciende significativamente, evidenciado en los promedios bajos obtenidos en las asignaturas con alto contenido de ciencias naturales y matemáticas. Estos resultados podrían atribuirse a una preparación, en el nivel precedente (específicamente en estas áreas) no acorde con los requerimientos de la universidad. En este sentido, en búsqueda de la articulación de los niveles educativos, la Educación Media debe ser el nivel de iniciación de la Educación Superior, que de forma sistemática y reflexiva se despliega en el campo del trabajo material e intelectual, y cuyo contenido se fundamenta en la formación científica, cultural y tecnológica (Calderón, 2006). En lo referente a la enseñanza de las Ciencias Naturales, podría decirse que muchas de las razones por las cuales los estudiantes encuentran las ciencias aburridas están relacionadas con las dificultades que se presentan en su enseñanza (Rabino, García, Moro \& Minnaard, 2002). La investigación educativa, ha permitido evidenciar diversos problemas asociados con la educación en Ciencias Naturales, tales como la falta de interés de los estudiantes, por el trabajo académico en este campo (Raya, 1997, citado en Jessup, Oviedo \& Castellanos, 2000), así como el aprendizaje memorístico, carente de autonomía y desarrollo progresivo, la presencia de esquemas de pensamiento pobres, rígidos y estereotipados que conducen al estancamiento, a la rutina y a una elaboración intelectual superficial o de bajo nivel cognoscitivo (Amestoy de Sánchez, 1993, citada en Jessup, Oviedo \& Castellanos, 2000).

Por otra parte, el conocimiento fundamental que adquieren los individuos sobre la naturaleza de la ciencia se da en el salón de clase, por lo tanto, lo que se enseña y se experimenta en el salón de clase de ciencia, estará basado o influenciado por el conocimiento que tenga el maestro sobre la naturaleza de ésta disciplina (Bybee, 1986, citado en Figueroa, 2003). En este mismo sentido Utria \& Figueroa (2003) afirman que los profesores universitarios de ciencias deben capacitarse con el fin de ofrecer un mejor proceso de enseñanza y aprendizaje que propenda por mejorar el nivel de comprensión conceptual de los estudiantes. 
Estudios han logrado establecer que los docentes de secundaria tienen un dominio insuficiente de sus disciplinas, de las concepciones de ciencia y de aprendizaje, pero especialmente del conocimiento científico (Flores, Gallegos, García, Vega \& García, 2003; Gallegos, Flores y Valdés, 2004; Chamizo, Nieto y Sosa, 2004, citados en Flores et al., 2007), lo que se traduce posteriormente en bajo rendimiento en los estudios superiores.

Se asume entonces la necesidad de profundas transformaciones que van desde los cambios en el enfoque del proceso de enseñanza aprendizaje, pasando por los cambios en el tipo de contenidos sujetos a evaluación hasta los cambios en la misma lógica de evaluación de los aprendizajes (Mateo \& Martínez, 2005, citados en Ortiz, 2006). Es indiscutible que la introducción de metodologías activas y centradas en el aprendizaje en ésta etapa preparatoria que debe ser la educación básica secundaria y media, se traducirán en una mejor preparación de los estudiantes para abordar sus estudios universitarios.

Sin embargo el logro de la convergencia deseada afronta entre otras dificultades, el poco acercamiento entre los docentes de los diferentes niveles y las dotaciones económicas y tecnológicas tan desiguales para el bachillerato con relación a la universidad (Ortiz, 2006) Pese a éstas y otras dificultades, bien vale la pena buscar la convergencia, ya que la articulación de ambos sistemas, se traduciría en mejores niveles educativos de las instituciones y consecuentemente universitarios de primer semestre con mejor formación y con herramientas de análisis y de aprendizaje autónomo que les permitan insertarse efectivamente en el trabajo universitario y obtener mejor rendimiento académico.

\section{Conclusiones}

El estudio permitió concluir que la mayoría de las egresadas del colegio El Divino Niño presentaron rendimiento académico regular en el nivel de Educación Media y una tendencia a mejorar el rendimiento académico en la universidad. Con los resultados se acepta la primera hipótesis alterna, de que si existe una relación estadísticamente positiva entre el rendimiento en Educación Media y la Trayectoria Escolar en la Universidad.

Con relación a los programas académicos de pregrado, puede afirmarse que las estudiantes con mejor trayectoria escolar cursan los programas de Negocios Internacionales, Derecho y Comunicación Social, Todas las estudiantes que cursan los programas de microbiología, ingeniería de petróleos, ingeniería de energía e ingeniería civil, obtuvieron trayectoria escolar alta y los programas en los que se registraron los mayores porcentajes de estudiantes con trayectorias escolares bajas fueron Marketing y Negocios Internacionales, Biología, Ingeniería Ambiental y Administración de Empresas.

Puede afirmarse que las estudiantes que optaron por programas relacionados con las ciencias naturales presentan un rendimiento satisfactorio en la universidad atendiendo que solo en los programas de Biología y de Ingeniería Ambiental hay estudiantes con trayectoria escolar baja.

- Existe una correlación muy débil entre el Rendimiento Académico en ciencias naturales y matemáticas en Educación Media y los promedios en las asignaturas relacionadas con estas áreas en la universidad, por lo cual se rechaza la segunda hipótesis alterna. En conclusión para las asignaturas del área de ciencias naturales la mayoría de las estudiantes obtienen promedios regulares, y para el caso de matemáticas la mayoría obtiene promedios bajos.

Si bien no puede afirmarse sea un predictor del rendimiento futuro, la consideración del mismo como factor de admisión en la universidad podría redundar en el beneficio de los estudiantes atendiendo a que las instituciones de los niveles precedentes y los mismos estudiantes deberían comprometerse con mejorar la educación ofrecida y el rendimiento académico respectivamente. 


\section{Bibliografía}

Addine, R. \& Ramírez, E. (2004). La influencia científico-cultural desde las ciencias naturales en el bachillerato cubano. Recuperado el 2 de septiembre de 2008, de http://www.redcientifica.com/doc/ doc200401210111.html

Andino, G., Cardozo, S., Duré, G., Falcon, M., Pierlorenzi, S., Gauna, M. y Markouusky, E. (2003). Caracterización y rendimiento de alumnos ingresantes a Medicina 2003 según el nivel de instrucción de los padres. Recuperado el 5 de junio de 2008 desde http://www.unne.edu.ar/Web/cyt/ cyt/2003/comunicaciones/09-Educacion/D024.pdf

Arias, F., Chávez, A. \& Muñoz I. (2006). El aprovechamiento previo y la escuela de procedencia como predictores del aprovechamiento futuro: Un caso. Revista Enseñanza e Investigación en Psicología, 11 (1), 5-22 [versión electrónica].

Barrantes, A \& Sequeira, A. (1993). Algunas causas que determinan el rendimiento académico, en matemática, de estudiantes de décimo y undécimo año en cuatro colegios de Costa Rica. Recuperado el 8 de septiembre de 2008 desde http://www. cimm.ucr.ac.cr/una/tesis/Algunas $\% 20$ causas $\% 20$ que $\% 20$ determinan $\% 20$ el $\% 20$ rendimiento $\% 20$ academico, $\% 20 \mathrm{en} \% 2$ 0Matematica, $\% 20 \mathrm{de} \% 20$ estudiantes $\% 20 \mathrm{de} \% 2$ 0decimo $\% 20 \mathrm{y} \% 20$ undecimo.pdf

Bastias, G., Villarroel, L., Zúñiga, D., Marshall, G., Velasco, N. \& Mena, B. (2000). Desempeño académico de los estudiantes de medicina. ¿Un resultado predecible? Revista Médica de Chile, 128 (6), 671-678 [versión electrónica].

Brito, R \& Amado M. (2007). Causas de reprobación en matemáticas en el Instituto
Tecnológico de Mexicali. Recuperado el 28 de octubre de 2008, desde http:// www.alammi.info/documentos/1 congreso/ memorias/jueve6_2.pdf

Cabrera, J. \& Fariñas, G. (s.f.). El estudio de los estilos de aprendizaje desde una perspectiva Vigostkiana: una aproximación conceptual Universidad de la Habana. Revista Iberoamericana de Educación, 37 (1) [versión electrónica: http://rieoei.org/delos lectores/1090Cabrera.pdf

Calderón, O. (2006). Articulación de la Educación Media en la perspectiva de la formación e ciencia y tecnología para la democratización del conocimiento. Proyecto Académico en Pedagogía de la Universidad Distrital Francisco José de Caldas. Recuperado el 5 de septiembre de 2008, de http://www.universia.net.co/ docentes/view-document/documento106.html

Cantú, I. (2004). El estilo de aprendizaje y la relación con el desempeño académico de los estudiantes de arquitectura de la UANL. Revista Ciencia UANL, 7, 72-79 [versión electrónica, recuperado el 25 de mayo de 2007, desde http://w3.dsi.uanl.mx/ publicaciones/ciencia-uanl/vol7/1/pdfs/ estudiantes_arquitectura.pdf]

Chaín, R., Cruz, N., Martínez, M., \& Jácome, N. (2003). Examen de Selección y Probabilidad de éxito escolar es Estudios Superiores. Estudio en una universidad pública estatal mexicana. Revista Electrónica de Investigación Educativa, 5 [versión electrónica, recuperado el 20 de junio de 2007, desde http://redie.ens.uabc.mx/ vol5nol/contenido-chain.html]

Cortés, A. \& Palomar, J. (2008). El proceso de admisión como predictor del rendimiento académico en la educación superior. Universitas Psychologica, 7, 199-215. 
Domínguez H. \& Pérez, M. (1993). El bachillerato, su evolución y su influencia en la demanda de carreras científicas en el nivel de licenciatura. Universidad Autónoma de México. Revista Perfiles Educativos. Recuperado el 30 de octubre de 2008 desde http://redalyc.uaemex.mx/redalyc/pdf/132/ 13206201.pdf

Duarte. M. \& Galaz, J. (2006). Predictores del Desempeño Académico en el Primer Año de Universidad en una Institución Pública Estatal. Recuperado el 30 de mayo de 2007, de: http://www.congresoretosyexpectativas.udg.mx/Congreso\%206/ Eje\%202/Ponencia_199.pdf

Duarte, M., Galaz, J. \& Rosales, J. (2005). Predictores de Desempeño Académico en una Cohorte al finalizar el 2 do y 5 to Semestre en una Universidad Pública Estatal. Recuperado el 30 de mayo de 2007, de: http://www.comie.org.mx/congreso/ memoria/v $9 /$ ponencias/at $16 /$ PRE1178912891.pdf

Edel, R. (2003). El rendimiento académico: concepto, investigación y desarrollo. Revista Electrónica Iberoamericana sobre Calidad, Eficacia y Cambio en Educación, 1(2) [versión electrónica: http://www.ice.deusto. es/rinace/reice/vol1n2/Edel.pdf]

Edel, R. (2003b). Factores asociados al rendimiento académico. Revista Iberoamericana de Educación, 12. Recuperado el 15 de mayo de 2007, desde www.rieoei. org/investigacion $1 . h t m$

Figueroa, R. (2003). Concepciones de los estudiantes universitarios sobre la naturaleza de la ciencia/conocimiento cientifico desde las perspectivas epistemológicas, empiristalpositivista y constructivista. Bogotá: Centro de InvestigacionesUniversidad Pedagógica Nacional.
Figueroa, N., Cataldi, Z., Méndez, P., Rendón, J.,Costa, G., Salgueiro, F., \& Lage, F. (2005). Los estilos de aprendizaje y el desgranamiento universitario en Carreras de informática. Facultad de Ingeniería. Universidad de Buenos Aires. Recuperado el 30 de octubre de 2008 desde http:// cs.uns.edu.ar/jeitics2005/Trabajos/pdf/ 03.pdf

Flores, F., Gallegos, L., García, A., Vega, E y García, V. (2007). El conocimiento de los profesores de ciencias naturales en secundaria: un estudio en tres niveles, centro de ciencias aplicadas y desarrollo tecnológico. UNAM. México. Revista Iberoamericana de Educación, 43 (3) [versión electrónica: www.rieoei.org/ 1800.htm]

García, F. \& Doménech, F. (1997). Motivación, aprendizaje y rendimiento escolar. Revista Electrónica de Motivación y Emoción, 1 [versión electrónica: http://reme.uji.es/ articulos/pa0001/texto.html]

García, M., Alvarado, J. \& Jiménez A. (2000). La predicción del rendimiento académico: regresión lineal versus regresión logística. Psicothema, 12, supl. 2, 248-252 [versión electrónica: http://www.psicothema.com/ pdf/558.pdf]

Gaviria, A. \& Barrientos, J. (2001). Determinantes de la calidad de la educación en Colombia. Departamento Nacional de Planeación Dirección de Estudios Económicos. Recuperado el 30 de octubre de 2008, desde http://ideas.repec.org/p/col/ 000118/002301.html

Herrera, F. (2004). ¿Cómo interactúan el autoconcepto y el rendimiento académico, en un contexto educativo pluricultural? Recuperado el 12 de agosto de 2008 desde http://www.rieoei.org/deloslectores/ 627Herrera.PDF 
Jessup, M., Oviedo, P. \& Castellanos, R. (2000). La resolución de problemas y la educación en ciencias naturales. Pedagogía y Saberes, 15 [versión electrónica http:// w3.pedagogica.edu.co/storage/ps/articulos/ pedysab15_07arti.pdf]

Martínez, V. \& Solís L. (2001). Selección estudiantil para el ingreso a la educación Superior, mediante el examen nacional EXANI -II y el rendimiento escolar del Nivel Medio Superior, en cuatro facultades de la Universidad Autónoma del Estado de México. Recuperado el 25 de mayo de 2007, desde http://www.congresoretos yexpectativas.udg.mx/Congreso $\% 202 /$ Mesa $\% 202 / \mathrm{c}) \% 20$ Evaluaci $\%$ F 3 n, $\% 20$ a creditaci $\%$ F 3 n $\% 20$ y $\% 20$ certificaci $\% \mathrm{~F} 3 \mathrm{n} / 2 . \mathrm{c} .4$..pdf

Ministerio de Educación Nacional. (2003). Situación de la Educación Media en Colombia. Recuperado el 20 de octubre de 2008, desde http://www.colombiaaprende. edu.co/html/mediateca/1607/article101871.html

Moler, E. (2008). Elección disciplinaria del sistema universitario argentino (Primera Parte): Un análisis sobre aspirantes (2006-2007-2008). Recuperado el 30 de octubre de 2008 desde http://www.me.gov. ar/spu/guia_tematica/estadisticas y_publicaciones/estaditicas_sobre_las preferencias.pdf

Morales, A. \& Arcos P. (1999). El entorno familiar y el rendimiento escolar. Recuperado el 25 de mayo de 2007, desde http://www.juntadeandalucia.es/averroes/ publicaciones/investigacion/entorno familia.pdf

Musayón, Y. (2001). Relación entre el Puntaje de Ingreso y el Rendimiento Académico en el Segundo año de las alumnas de Enfermería ingresantes entre los años 1994
- 1997 en una Universidad Peruana. Revista Universidades, 22. Recuperado el 5 de junio de 2007 desde http://www.udual.org/ CIDU/Revista/22/RelaEnfermeria.htm.

Noriega, P. (1989). El Rendimiento escolar en Ciencias Básicas y su mejoramiento a través de condiciones de estudio apropiadas para el alumno. Recuperado el 30 de octubre de 2008 desde http:// www.anuies.mx/servicios/p_anuies/ publicaciones/revsup/res070/txt15.htm

Núñez, J., Steiner, R., Cadena, X. \& Pardo, R. (2002). ¿Cuáles colegios ofrecen mejor educación en Colombia?. Recuperado el 30 de octubre de 2008 desde http:// www.webpondo.org/files/opinion jul_sep_2002/NU\%D1EZSTEINER CUALES ONLOSCOLEGIOS.pdf

Ortiz, M. (2006). El Espacio Europeo de Educación Superior y el Bachillerato: Oportunidades y Retos para la Orientación. Recuperado el 7 de septiembre de 2008 desde http:/www.upv.es/cies2006/ documentos/U0316440.pdf

Porto, A., Di Gresia, L. \& López, M. (2005). Admisión a la Universidad y rendimiento de los estudiantes. Recuperado el 25 de junio de 2007, desde http://www.depeco. econo.unlp.edu.ar/espec/admision 2004b.pdf

Rabino, M., García, M., Moro, M. \& Minnaard, V. (2002). Una propuesta para secuencias los contenidos en Ciencias Naturales desde la perspectiva Lakatosiana. Revista Iberoamericana de Educación [versión electrónica: http://www.rieoei.org/delos lectores/317Rabino.pdf]

Reyes, Y. (2003). Relación entre el rendimiento académico, la ansiedad ante los exámenes, los rasgos de personalidad, el autoconcepto y la asertividad en estudiantes del primer 
año de Psicología de la UNMSM. Tesis de pregrado, Universidad Nacional Mayor de San Marcos, Perú. [versión electrónica: http://sisbib. unmsm.edu.pe/Bibvirtual/tesis/ Salud/Reyes_T_Y/Reyes_T_Y.htm]

Robles, R. (2004). Orientación Educativa y Rendimiento Académico. Revista Mexicana de Orientación Educativa, 2. [versión electrónica: http://www.remo.ws/ revista/n4/n4-robles.htm].

Rojas, L. (2005). Influencia del Entorno Familiar en el Rendimiento Académico de Niños y Niñas con Diagnóstico de Maltrato de la Escuela de Calarcá de Ibagué. Tesis de especialización, Pontificia Universidad Javeriana, Bogotá, Colombia.

Tirado, F., Backhoff; E., Larrazolo, N. \& Rojas, M. (1997). Validez predictiva del Examen de Habilidades y Conocimientos Básicos (EXCHOBA). Revista Mexicana de Investigación Educativa, 2 (3), 67-84 [versión electrónica: http://www.comie. org. $\mathrm{mx} / \mathrm{v} 1 / \mathrm{revista} / \mathrm{visualizador}$.php? articulo=ART00213\&criterio $=$ http: $/ /$ www.comie.org.mx/documentos/rmie/v02/ n003/pdf/rmiev02n03scC00n04es.pdf]

Torres, R. (1996). Formación docente, clave de la reforma Nuevas formas de aprender $y$ enseñar, UNESCO-OREALC. Recuperado el 30 de octubre de 2008, desde http://www. fronesis.org
Utria, C. \& Figueroa, R. (2003). Entendimiento del concepto Mol logrado por los estudiantes en un curso de química general de corte constructivista. Recuperado el 28 de octubre de 2008, desde http://w3. pedagogica.edu.co/storage/ted/articulos/ ted13_04arti.pdf

Valderrama, G. (2001). Validez ligada a criterio de los factores de Admisión a la Escuela de Psicología de la Universidad Católica Santa María la Antigua. Recuperado el 8 de septiembre de 2008 desde http://www. usma.ac.pa/web/DI/images/cipsu/INFORME $\% 20$ FINAL $\% 20$ PREDICCI $\%$ C3\%93N.pdf

Valle, A., González, R., Núñez, J., Suárez, J., Piñeiro, I. \& Rodríguez, S. (2000). Enfoques de aprendizaje en estudiantes universitarios. Psicothema, 12 (3), 368-375 [versión electrónica: http://www.psicothema.com/ pdf/344.pdf].

Vélez, A y Roa, C. (2005). Factores Asociados al Rendimiento Académico en estudiantes de medicina. Eduación Médica, 8 (2), 7482 [versión electrónica: http://scielo.isciii.es/ pdf/edu/v8n2/original1.pdf]

Viloria, J. (2006). Educación Superior en el Caribe Colombiano. Recuperado el 20 de octubre de 2008 desde http://www.banrep. gov.co/documentos/publicaciones/regional/ documentos/DTSER-69\%20(VE).pdf 
